\title{
Operator-valued version of conditionally free product
}

\author{
by \\ Wojciech MєotKowski (Wrocław)
}

\begin{abstract}
We present an operator-valued version of the conditionally free product of states and measures, which in the scalar case was studied by Bożejko, Leinert and Speicher. The related combinatorics and limit theorems are provided.
\end{abstract}

1. Introduction. The concept of free probability has been developed since the pioneering work of Voiculescu [V]. In this theory a probability space is a unital complex $*$-algebra $\mathcal{A}$, elements of which are viewed as random variables, endowed with a state $\phi$ which plays the role of the expectation. Having a family $\left(\mathcal{A}_{i}, \phi_{i}\right)_{i \in I}$ of such probability spaces we can define another one, $(\mathcal{A}, \phi)$, where $\mathcal{A}$ is the unital free product $*_{i \in I} \mathcal{A}_{i}$ and $\phi$ is a state defined by the property that $\phi\left(a_{1} \ldots a_{m}\right)=0$ whenever $a_{1} \in \mathcal{A}_{i_{1}}, \ldots, a_{m} \in \mathcal{A}_{i_{m}}$, $i_{1} \neq \ldots \neq i_{m}$ and $\phi_{i_{1}}\left(a_{1}\right)=\ldots=\phi_{i_{m}}\left(a_{m}\right)=0$. In this situation one says that the family $\left\{\mathcal{A}_{i}\right\}_{i \in I}$ of subalgebras is free in the probability space $(\mathcal{A}, \phi)$. This notion leads naturally to that of free convolution of probability measures on the real line. Speicher [S2, S3] has provided a combinatorial description of this theory by using the lattice of noncrossing partitions.

Later on Bożejko, Leinert and Speicher [BS, BLS] investigated probability spaces $\mathcal{A}$ endowed with a pair $(\phi, \psi)$ of states. Having a family $\left(\mathcal{A}_{i}, \phi_{i}, \psi_{i}\right)_{i \in I}$ of such spaces they were able to construct a probability space $(\mathcal{A}, \phi, \psi)$ such that $\mathcal{A}=*_{i \in I} \mathcal{A}_{i}$ and $\psi\left(a_{1} \ldots a_{m}\right)=0, \phi\left(a_{1} \ldots a_{m}\right)=$ $\phi_{i_{1}}\left(a_{1}\right) \ldots \phi_{i_{m}}\left(a_{m}\right)$ whenever $a_{1} \in \mathcal{A}_{i_{1}}, \ldots, a_{m} \in \mathcal{A}_{i_{m}}, i_{1} \neq \ldots \neq i_{m}$, $\psi_{i_{1}}\left(a_{1}\right)=\ldots=\psi_{i_{m}}\left(a_{m}\right)=0$ (conditional freeness of the family $\left\{\mathcal{A}_{i}\right\}_{i \in I}$ in $(\mathcal{A}, \phi, \psi))$. This led them to the notion of free convolution of pairs of probability measures on $\mathbb{R}$.

Here we are going to extend this theory to the situation when $\phi$ is allowed to be an operator-valued state, i.e. when $\phi$ is of the form $\phi(a)=\left.P_{0} \pi(a)\right|_{\mathcal{H}_{0}}$, where $\mathcal{H}_{0}$ is a fixed Hilbert space, $\pi$ is a $*$-representation of $\mathcal{A}$ in a Hilbert space $\mathcal{H} \supset \mathcal{H}_{0}$ and $P_{0}$ is the orthonormal projection of $\mathcal{H}$ onto $\mathcal{H}_{0}$, so that

2000 Mathematics Subject Classification: 46L54, 46L09, 43A65.

Research supported by KBN grant 2 PO3A 05415. 
$\phi$ is a completely positive function $\mathcal{A} \rightarrow \mathcal{B}\left(\mathcal{H}_{0}\right)$. We introduce this notion in order to study conditionally free convolution of operator-valued measures (see for example the papers of Bisgaard [Bi] and Schmüdgen [Sm] and the references given there). In particular we extend the boolean convolution of measures, studied by Speicher and Woroudi [SW], to operator-valued measures. Note that neither the classical nor the free convolution of probability measures admits such an operator-valued version. It is also posible to consider spaces with more than two states (see [M2]) but then the related product of states and measures is no longer associative and for this reason the methods presented here are not applicable.

It is confusing that the term "operator-valued free probability" appears also in literature with a different meaning (see $[\mathrm{S} 3, \mathrm{Sh}]$ ). There a probability space is a triple $(\mathcal{B}, \mathcal{A}, \phi)$, where $\mathcal{A}$ is a unital algebra, $\mathcal{B}$ is its subalgebra with $1 \in \mathcal{B}$, and $\phi: \mathcal{A} \rightarrow \mathcal{B}$ satisfies: $\phi(b)=b$ for $b \in \mathcal{B}$, and $\phi\left(b_{1} a b_{2}\right)=b_{1} \phi(a) b_{2}$ for $a \in \mathcal{A}, b_{1}, b_{2} \in \mathcal{B}$, i.e. $\phi$ is a $\mathcal{B}$-functional. The distribution of an element $a \in \mathcal{A}$ is a $\mathcal{B}$-functional $\nu_{a}$ defined on the algebra $\mathcal{B}\langle X\rangle$ generated freely by $\mathcal{B}$ and an indeterminate $X$ such that $\nu_{a}(P(X)):=\phi(P(a))$ for $P(X) \in \mathcal{B}\langle X\rangle$. The natural objects to study in this setup are amalgamated free products.

The paper is organized as follows. First we study the notions of a moment pair and a cumulant pair of functions on a fixed linear subspace $\mathcal{A}^{0}$ of a unital algebra $\mathcal{A}$, with $1 \in \mathcal{A}^{0}$, which are analogous to those of moment and cumulant functions discussed by Speicher [S2, S3]. In Proposition 1 we show that these two notions are in one-to-one correspondence. The difference compared with the case studied by Speicher is that dealing with pairs one has to distinguish between inner and outer blocks of noncrossing partitions. Although these notions appeared in [BLS], they were not studied systematically there. Here we provide more details and adapt this to the situation when the first functions in the pairs are operator-valued ones.

Then we apply our results to triples of the form $(\mathcal{A}, \Phi, \psi)$, where $\mathcal{A}$ is a unital algebra over a field $K, \psi: \mathcal{A} \rightarrow K$ is a linear functional satisfying $\psi(1)=1$ and $\Phi$ is a linear function $\mathcal{A} \rightarrow \mathcal{B}$, where $\mathcal{B}$ is a fixed algebra over $K$ with a unit $\mathbf{1}$, satisfying $\Phi(1)=\mathbf{1}$. For a family $\{(\mathcal{A}, \Phi, \psi)\}_{i \in I}$ of such triples we construct another one, $(\mathcal{A}, \Phi, \psi)$, which satisfies:

1) $\mathcal{A}=*_{i \in I} \mathcal{A}_{i}$,

2) $\psi\left(a_{1} \ldots a_{m}\right)=0$ and $\Phi\left(a_{1} \ldots a_{m}\right)=\Phi_{i_{1}}\left(a_{1}\right) \ldots \Phi_{i_{m}}\left(a_{m}\right)$ whenever $a_{1} \in \mathcal{A}_{i_{1}}, \ldots, a_{m} \in \mathcal{A}_{i_{m}}, i_{1} \neq \ldots \neq i_{m}$ and $\psi_{i_{1}}\left(a_{1}\right)=\ldots=\psi_{i_{m}}\left(a_{m}\right)=0$.

We show in Proposition 2 that this construction is associative.

Next, assuming that $K=\mathbb{C}$ and that $\mathcal{B}$ is endowed with a norm, we study the limit theorems providing operator-valued versions of the central limit theorem and of the Poisson distribution (cf. [S1, BS, BLS]). 
In Section 6 we prove that the conditionally free product $(\mathcal{A}, \Phi, \psi)=$ $*_{i \in I}\left(\mathcal{A}_{i}, \Phi_{i}, \psi_{i}\right)$ preserves complete positivity of the states $\Phi$ and $\psi$. The construction of the related pair of representations is adapted from [M1] where it was studied for the group case (see also [B1, B2, B3]).

Finally we discuss conditionally free convolution on the class $\mathcal{M}(\mathcal{H}) \times \mathcal{M}$, where $\mathcal{M}$ (resp. $\mathcal{M}(\mathcal{H})$ ) denotes the class of all (resp. all operator-valued) compactly supported probability measures on $\mathbb{R}$. As a consequence we obtain a binary associative and commutative operation $\uplus$ on $\mathcal{M}(\mathcal{H})$ which extends the boolean convolution on $\mathcal{M}$ introduced by Speicher and Woroudi [SW]. We show in particular that if $E$ (resp. $F$ ) is the spectral measure of a selfadjoint operator $A$ (resp. $B$ ) then $E \uplus F$ is the spectral measure of $A+B$.

2. Preliminaries. By a partition of a set $S$ we mean a family $\pi$ of nonempty, pairwise disjoint subsets of $S$ (called blocks) such that $\bigcup \pi=S$. The corresponding equivalence relation on $S$ will be denoted by $\stackrel{\pi}{\sim}$.

A partition $\pi$ of $\{1, \ldots, m\}$ is called noncrossing if $1 \leq k<p<l<q \leq m$, $k, l \in V \in \pi, p, q \in W \in \pi$ implies $V=W$. The class of all noncrossing partitions of $\{1, \ldots, m\}$ will be denoted by $\mathrm{NC}(m)$. A block $V \in \pi \in \mathrm{NC}(m)$ is said to be inner if there is another block $W \in \pi$ and elements $p, q \in W$ such that $p<k<q$ for every $k \in V$. Otherwise $V$ is called outer. The family of all inner (resp. outer) blocks of a noncrossing partition $\pi$ will be denoted by $\pi_{\mathrm{i}}\left(\right.$ resp. $\pi_{\mathrm{o}}$ ). Having a product of the form $\prod_{V \in \pi_{\mathrm{o}}} f(V)$ we will assume that the factors are in the same order as the blocks in $\pi_{\mathrm{o}}$.

For $\pi \in \mathrm{NC}(m)$ and $1 \leq p<m$ we define $\pi(p=p+1)$ as the partition $\sigma \in \mathrm{NC}(m-1)$ which results from $\pi$ by identifying $p$ and $p+1$ and joining the blocks containing them, if they are different (see Remark 3.2.2 in [S3]).

In other words, if for a block $U=\left\{k_{1}, \ldots, k_{s}\right\}$ with $k_{1}<\ldots<k_{r} \leq p<$ $k_{r+1}<\ldots<k_{s}$, we define $U(p):=\left\{k_{1}, \ldots, k_{r}, k_{r+1}-1, \ldots, k_{s}-1\right\}$ then $\sigma=\pi(p=p+1)$ consists of blocks $U(p)$ with $p, p+1 \notin U \in \pi$ and the block $U^{\prime}(p) \cup U^{\prime \prime}(p)$, where $p \in U^{\prime} \in \pi$ and $p+1 \in U^{\prime \prime} \in \pi$ (possibly $U^{\prime}=U^{\prime \prime}$ ). Note that if $\pi$ is noncrossing then so is $\pi(p=p+1)$.

For a sequence $\mathbf{a}=\left(a_{1}, \ldots, a_{m}\right)$ and a set $V=\left\{k_{1}, \ldots, k_{s}\right\}$ of integers with $1 \leq k_{1}<\ldots<k_{s} \leq m$, we define the subsequence $\mathbf{a}(V)=$ $\left(a_{1}, \ldots, a_{m}\right)(V):=\left(a_{k_{1}}, \ldots, a_{k_{s}}\right)$ and the product $\prod_{k \in V} a_{k}:=a_{k_{1}} \ldots a_{k_{s}}$.

3. Moment and cumulant pairs of functions. The notions of moment and cumulant functions were introduced by Speicher [S3, Definition 3.2.1] as a tool to calculate expressions of the form $\phi\left(a_{1} \ldots a_{m}\right)$, where $\phi=*_{i \in I} \phi_{i}$ is the free product state on a free product algebra $\mathcal{A}=*_{i \in I} \mathcal{A}_{i}$ and $a_{1} \in \mathcal{A}_{i_{1}}, \ldots, a_{m} \in \mathcal{A}_{i_{m}}$. Our aim is to extend them to moment and cumulant pairs in order to deal with the conditionally free product pair of states $(\phi, \psi)=*_{i \in I}\left(\phi_{i}, \psi_{i}\right)$. 
Throughout this section $\mathcal{A}$ will be a fixed unital algebra over a field $K$ and $\mathcal{A}^{0}$ will denote a linear subspace of $\mathcal{A}$ with $1 \in \mathcal{A}^{0}$. In our applications $\mathcal{A}^{0}$ will be either the whole $\mathcal{A}$ or the linear span $\operatorname{lin}\left\{\mathcal{A}_{i}: i \in I\right\}$ in the free product $*_{i \in I} \mathcal{A}_{i}$. Also, $\mathcal{B}$ will be a fixed algebra over $K$ with a unit $\mathbf{1}$. We will assume that $f, F, r, R$ are functions

$$
f, r: \bigcup_{m=1}^{\infty} \underbrace{\mathcal{A}^{0} \times \ldots \times \mathcal{A}^{0}}_{m \text { times }} \rightarrow K, \quad F, R: \bigcup_{m=1}^{\infty} \underbrace{\mathcal{A}^{0} \times \ldots \times \mathcal{A}^{0}}_{m \text { times }} \rightarrow \mathcal{B}
$$

such that $f, F, r, R$ restricted to $\mathcal{A}^{0} \times \ldots \times \mathcal{A}^{0}$ ( $m$ times) are $m$-linear for every $m$.

Definition. The pair $(F, f)$ will be called a moment pair of functions on $\mathcal{A}^{0}$ if $f(1)=1, F(1)=\mathbf{1}$ and

$$
\begin{aligned}
& f\left(a_{1}, \ldots, a_{p-1}, a_{p} a_{p+1}, a_{p+2}, \ldots, a_{m}\right) \\
&= f\left(a_{1}, \ldots, a_{p-1}, a_{p}, a_{p+1}, a_{p+2}, \ldots, a_{m}\right), \\
& F\left(a_{1}, \ldots, a_{p-1}, a_{p} a_{p+1}, a_{p+2}, \ldots, a_{m}\right) \\
&=
\end{aligned}
$$

whenever $1 \leq p<m, a_{1}, \ldots, a_{m}, a_{p} a_{p+1} \in \mathcal{A}^{0}$.

The pair $(R, r)$ will be called a cumulant pair of functions on $\mathcal{A}^{0}$ if $r(1)=1, R(1)=\mathbf{1}$,

$$
\begin{aligned}
& r\left(a_{1}, \ldots, a_{p-1}, a_{p} a_{p+1}, a_{p+2}, \ldots, a_{m}\right) \\
& =r\left(a_{1}, \ldots, a_{p}, a_{p+1}, \ldots, a_{m}\right)+r\left(a_{1}, \ldots, a_{p}\right) r\left(a_{p+1}, \ldots, a_{m}\right) \\
& \quad+\sum_{k=2}^{p} r\left(a_{k}, \ldots, a_{p}\right) r\left(a_{1}, \ldots, a_{k-1}, a_{p+1}, \ldots, a_{m}\right) \\
& \quad+\sum_{k=p+1}^{m-1} r\left(a_{p+1}, \ldots, a_{k}\right) r\left(a_{1}, \ldots, a_{p}, a_{k+1}, \ldots, a_{m}\right),
\end{aligned}
$$

and

(1b)

$$
\begin{aligned}
& R\left(a_{1}, \ldots, a_{p-1}, a_{p} a_{p+1}, a_{p+2}, \ldots, a_{m}\right) \\
& =R\left(a_{1}, \ldots, a_{p}, a_{p+1}, \ldots, a_{m}\right)+R\left(a_{1}, \ldots, a_{p}\right) R\left(a_{p+1}, \ldots, a_{m}\right) \\
& \quad+\sum_{k=2}^{p} r\left(a_{k}, \ldots, a_{p}\right) R\left(a_{1}, \ldots, a_{k-1}, a_{p+1}, \ldots, a_{m}\right) \\
& \quad+\sum_{k=p+1}^{m-1} r\left(a_{p+1}, \ldots, a_{k}\right) R\left(a_{1}, \ldots, a_{p}, a_{k+1}, \ldots, a_{m}\right)
\end{aligned}
$$

whenever $1 \leq p<m, a_{1}, \ldots, a_{m}, a_{p} a_{p+1} \in \mathcal{A}^{0}$. 
Note that the right hand sides are sums over all noncrossing partitions $\sigma$ of $\{1, \ldots, m\}$ such that $\sigma(p=p+1)$ is the one-block partition $\{\{1,2, \ldots$, $m-1\}\}$. In (1b) we put $R$ if the corresponding block of $\sigma$ is outer. In particular $r\left(a_{1} a_{2}\right)=r\left(a_{1}, a_{2}\right)+r\left(a_{1}\right) r\left(a_{2}\right)$ and $R\left(a_{1} a_{2}\right)=R\left(a_{1}, a_{2}\right)+R\left(a_{1}\right) R\left(a_{2}\right)$ if $a_{1}, a_{2}, a_{1} a_{2} \in \mathcal{A}^{0}$.

A single function $f$ (resp. $r$ ) is said to be a moment (resp. cumulant) function on $\mathcal{A}^{0}$ if $(f, f)$ (resp. $(r, r)$ ) is a moment (resp. cumulant) pair on $\mathcal{A}^{0}$.

ExAmples. 1) If $\mathcal{A}^{0}$ is a subalgebra of $\mathcal{A}$ then

$$
f\left(a_{1}, \ldots, a_{m}\right)=f\left(a_{1} \ldots a_{m}\right) \text { and } F\left(a_{1}, \ldots, a_{m}\right)=F\left(a_{1} \ldots a_{m}\right) .
$$

2 ) Assume that $r\left(a_{1}, \ldots, a_{m}\right)=0$ whenever $m \geq 2$. Then $r$ is a cumulant function if and only if the map $a \mapsto r(a)$ satisfies $r\left(a_{1} a_{2}\right)=r\left(a_{1}\right) r\left(a_{2}\right)$ whenever $a_{1}, a_{2}, a_{1} a_{2} \in \mathcal{A}_{0}$. In view of the next proposition, such a cumulant function corresponds to the moment function given by $f\left(a_{1}, a_{2}, \ldots, a_{m}\right):=$ $r\left(a_{1}\right) r\left(a_{2}\right) \ldots r\left(a_{m}\right)$.

3) If $r \equiv 0$ then $R$ becomes a boolean cumulant (see [SW, M2]).

We now show that these two notions are in one-to-one correspondence. Note that the first statement is contained in [S2, S3].

Proposition 1. Suppose that the pairs $(F, f)$ and $(R, r)$ of functions are related by

$$
f\left(a_{1}, \ldots, a_{m}\right)=\sum_{\pi \in \mathrm{NC}(m)} \prod_{V \in \pi} r\left(\left(a_{1}, \ldots, a_{m}\right)(V)\right)
$$

and

$$
F\left(a_{1}, \ldots, a_{m}\right)=\sum_{\pi \in \mathrm{NC}(m)} \prod_{V \in \pi_{\mathrm{i}}} r\left(\left(a_{1}, \ldots, a_{m}\right)(V)\right) \prod_{V \in \pi_{\mathrm{o}}} R\left(\left(a_{1}, \ldots, a_{m}\right)(V)\right),
$$

where the order of factors in the product $\prod_{V \in \pi_{\mathrm{o}}} R\left(\left(a_{1}, \ldots, a_{m}\right)(V)\right)$ corresponds to that of the outer blocks in $\pi$. Then

(a) $f$ is a moment function if and only if $r$ is a cumulant function.

(b) $(F, f)$ is a moment pair if and only if $(R, r)$ is a cumulant pair.

Proof. Put $\mathbf{a}=\left(a_{1}, \ldots, a_{m}\right)$ and $\mathbf{a}_{p}=\left(a_{1}, \ldots, a_{p} a_{p+1}, \ldots, a_{m}\right)$. First observe that if $(R, r)$ is a cumulant pair, $1 \leq p<m, a_{1}, \ldots, a_{m}, a_{p} a_{p+1} \in \mathcal{A}^{0}$, $\sigma \in \mathrm{NC}(m-1)$ then

$$
\prod_{B \in \sigma} r\left(\mathbf{a}_{p}(B)\right)=\sum_{\substack{\pi \in \mathrm{NC}(m) \\ \pi(p=p+1)=\sigma}} \prod_{V \in \pi} r(\mathbf{a}(V))
$$

and 
$(2 \mathrm{~b})$

$$
\begin{aligned}
\prod_{B \in \sigma_{\mathrm{i}}} r\left(\mathbf{a}_{p}(B)\right) \prod_{B \in \sigma_{\mathrm{o}}} R\left(\mathbf{a}_{p}(B)\right) & \\
& =\sum_{\substack{\pi \in \mathrm{NC}(m) \\
\pi(p=p+1)=\sigma}} \prod_{V \in \pi_{\mathrm{i}}} r(\mathbf{a}(V)) \prod_{V \in \pi_{\mathrm{o}}} R(\mathbf{a}(V)) .
\end{aligned}
$$

Therefore

$$
\begin{aligned}
F\left(\mathbf{a}_{p}\right) & =\sum_{\sigma \in \mathrm{NC}(m-1)} \prod_{B \in \sigma_{\mathrm{i}}} r\left(\mathbf{a}_{p}(B)\right) \prod_{B \in \sigma_{\circ}} R\left(\mathbf{a}_{p}(B)\right) \\
& =\sum_{\sigma \in \mathrm{NC}(m-1)} \sum_{\substack{\pi \in \mathrm{NC}(m) \\
\pi(p=p+1)=\sigma}} \prod_{V \in \pi_{\mathrm{i}}} r(\mathbf{a}(V)) \prod_{V \in \pi_{\circ}} R(\mathbf{a}(V)) \\
& =\sum_{\pi \in \mathrm{NC}(m)} \prod_{V \in \pi_{\mathrm{i}}} r(\mathbf{a}(V)) \prod_{V \in \pi_{\circ}} R(\mathbf{a}(V))=F(\mathbf{a}),
\end{aligned}
$$

and similarly for $f$, so that $(F, f)$ is a moment pair.

On the other hand, if $(F, f)$ is a moment pair then $R(1)=F(1)=\mathbf{1}$ and for $a_{1}, a_{2}, a_{1} a_{2} \in \mathcal{A}^{0}$,

$$
R\left(a_{1} a_{2}\right)=F\left(a_{1} a_{2}\right)=F\left(a_{1}, a_{2}\right)=R\left(a_{1}, a_{2}\right)+R\left(a_{1}\right) R\left(a_{2}\right)
$$

and similarly $r\left(a_{1} a_{2}\right)=r\left(a_{1}\right) r\left(a_{2}\right)$, so (1) holds for $m=2$. Assume that $m \geq 3$ and that (1) is true for all $k<m$. Then (2) holds for all $\sigma \in$ $\mathrm{NC}(m-1) \backslash\{\{1, \ldots, m-1\}\}$ so if $1 \leq p<m, a_{1}, \ldots, a_{m}, a_{p} a_{p+1} \in \mathcal{A}^{0}$ then

$$
\begin{aligned}
R\left(\mathbf{a}_{p}\right) & =F\left(\mathbf{a}_{p}\right)-\sum_{\substack{\sigma \in \mathrm{NC}(m-1) \\
|\sigma|>1}} \prod_{B \in \sigma_{\mathrm{i}}} r\left(\mathbf{a}_{p}(B)\right) \prod_{B \in \sigma_{\circ}} R\left(\mathbf{a}_{p}(B)\right) \\
& =F(\mathbf{a})-\sum_{\substack{\sigma \in \mathrm{NC}(m-1) \\
|\sigma|>1}} \sum_{\substack{\pi \in \mathrm{NC}(m) \\
\pi(p=p+1)=\sigma}} \prod_{V \in \pi_{\mathrm{i}}} r(\mathbf{a}(V)) \prod_{V \in \pi_{\circ}} R(\mathbf{a}(V)) \\
& =F(\mathbf{a})-\sum_{\substack{\pi \in \mathrm{NC}(m) \\
|\pi(p=p+1)|>1}} \prod_{V \in \pi_{\mathrm{i}}} r(\mathbf{a}(V)) \prod_{V \in \pi_{\circ}} R(\mathbf{a}(V)) \\
& =\sum_{\substack{|\in \mathrm{NC}(m)\\
| \pi(p=p+1) \mid=1}} \prod_{V \in \pi_{\mathrm{i}}} r(\mathbf{a}(V)) \prod_{V \in \pi_{\circ}} R(\mathbf{a}(V)),
\end{aligned}
$$

which is the right hand side of $(1 \mathrm{~b})$. The same works for $r$, so our proof is complete.

EXAMPLE. If $r \equiv 0$ then the formula for $F$ becomes

$$
F\left(a_{1}, \ldots, a_{m}\right)=\sum_{\substack{\pi \in \mathrm{NC}(m) \\ \pi_{\mathrm{i}}=\emptyset}} \prod_{V \in \pi} R\left(\left(a_{1}, \ldots, a_{m}\right)(V)\right),
$$


which can be inverted ([SW, M2]) to

$$
R\left(a_{1}, \ldots, a_{m}\right)=\sum_{\substack{\pi \in \mathrm{NC}(m) \\ \pi_{\mathrm{i}}=\emptyset}}(-1)^{|\pi|-1} \prod_{V \in \pi} F\left(\left(a_{1}, \ldots, a_{m}\right)(V)\right) .
$$

The following lemma will allow us to apply these notions to the unital free product of algebras.

Lemma 1. Assume that $(R, r)$ is a cumulant pair.

(a) If $m \geq 2, a_{1}, \ldots, a_{m} \in \mathcal{A}^{0}$ and $a_{p}=1$ for some $p$, with $1 \leq p \leq m$, then $R\left(a_{1}, \ldots, a_{m}\right)=0$ and $r\left(a_{1}, \ldots, a_{m}\right)=0$.

(b) Suppose that $m \geq 2, a_{1}, \ldots, a_{m}, b_{1}, \ldots, b_{m} \in \mathcal{A}^{0}$ and $a_{1}-b_{1}, \ldots$, $a_{m}-b_{m} \in K 1$. Then $R\left(a_{1}, \ldots, a_{m}\right)=R\left(b_{1}, \ldots, b_{m}\right)$ and $r\left(a_{1}, \ldots, a_{m}\right)=$ $r\left(b_{1}, \ldots, b_{m}\right)$.

Proof. For $m=2$ we have

$$
R(a)=R(a 1)=R(a, 1)+R(a) R(1)=R(a, 1)+R(a)
$$

so $R(a, 1)=0$. Similarly $R(1, a)=0$ and the same is true for $r$.

If the assertion holds for all $k<m$ then by definition $R\left(a_{1}, \ldots, 1 a_{p}, \ldots, a_{m}\right)=R\left(a_{1}, \ldots, 1, a_{p}, \ldots, a_{m}\right)+r(1) R\left(a_{1}, \ldots, a_{p}, \ldots, a_{m}\right)$ $(r(1)=1$ should be replaced by $R(1)=\mathbf{1}$ if $p=1)$, and similarly for $r$, which concludes the proof of the first part. The second assertion is an easy consequence of the first one.

Lemma 2. Suppose that functions $f$ and $r$ are related as in Proposition $1, \sigma=\left\{B_{1}, \ldots, B_{r}\right\}=\pi_{\mathrm{o}}$ for some $\pi \in \mathrm{NC}(m), B=B_{1} \cup \ldots \cup B_{r}=$ $\left\{k_{0}, k_{1}, \ldots, k_{s}\right\}, 1=k_{0}<k_{1}<\ldots<k_{s}=m$. Then

$$
\sum_{\substack{\pi \in \mathrm{NC}(m) \\ \pi_{\mathrm{o}}=\sigma}} \prod_{V \in \pi_{\mathrm{i}}} r\left(\left(a_{1}, \ldots, a_{m}\right)(V)\right)=\prod_{t=1}^{s} f\left(a_{k_{t-1}+1}, a_{k_{t-1}+2}, \ldots, a_{k_{t}-1}\right) .
$$

Proof. This is a consequence of the fact that $\left\{\pi \in \mathrm{NC}(m): \pi_{\mathrm{o}}=\sigma\right\}$ is precisely the class of partitions of the form $\sigma \cup \pi^{1} \cup \ldots \cup \pi^{s}$, where $\pi^{t}$ is a noncrossing partition of the interval $\left\{k_{t-1}+1, k_{t-1}+2, \ldots, k_{t}-1\right\}$.

4. Free product of pairs of functions. Here we will work with a family $\left\{\mathcal{A}_{i}\right\}_{i \in I}$ of unital algebras over $K$. Assume that for every $i \in I$ we are given linear functions $\Phi_{i}: \mathcal{A}_{i} \rightarrow \mathcal{B}, \psi_{i}: \mathcal{A}_{i} \rightarrow K$ such that $\Phi_{i}(1)=\mathbf{1}$ and $\psi_{i}(1)=1$. We define a moment pair

$$
F_{i}\left(a_{1}, \ldots, a_{m}\right)=\Phi_{i}\left(a_{1} \ldots a_{m}\right), \quad f_{i}\left(a_{1}, \ldots, a_{m}\right)=\psi_{i}\left(a_{1} \ldots a_{m}\right)
$$

on $\mathcal{A}_{i}$ and take the corresponding cumulant pair $\left(R_{i}, r_{i}\right)$. 
Now define $\mathcal{A}$ to be the unital free product $\mathcal{A}=*_{i \in I} \mathcal{A}_{i}$ and put $\mathcal{A}^{0}=$ $\operatorname{lin}\left\{\mathcal{A}_{i}: i \in I\right\}$ (note that in [S2, S3, BLS] the symbol "lin" is missing). We define

$$
R\left(a_{1}, \ldots, a_{m}\right)=\sum_{i \in I} R_{i}\left(a_{1}^{i}, \ldots, a_{m}^{i}\right), \quad r\left(a_{1}, \ldots, a_{m}\right)=\sum_{i \in I} r_{i}\left(a_{1}^{i}, \ldots, a_{m}^{i}\right),
$$

if $a_{k}=\sum_{i \in I} a_{k}^{i}, a_{k}^{i} \in \mathcal{A}_{i}$, and all but finitely many of the summands are 0 . Note that if $a=\sum_{i \in I} a^{i}=\sum_{i \in I} b^{i}, a^{i}, b^{i} \in \mathcal{A}_{i}$ then $b^{i}=a^{i}+\alpha^{i} 1$ for some $\alpha^{i} \in K$ satisfying $\sum_{i \in I} \alpha_{i}=0$. Therefore, in view of Lemma $1 \mathrm{~b}$, the functions $R$ and $r$ are well defined and one can see immediately

Lemma 3. Suppose that $a_{1} \in \mathcal{A}_{i_{1}}, \ldots, a_{m} \in \mathcal{A}_{i_{m}}$ and $i_{k} \neq i_{l}$ for some $1 \leq k<l \leq m$. Then $R\left(a_{1}, \ldots, a_{m}\right)=0$ and $r\left(a_{1}, \ldots, a_{m}\right)=0$.

Now we can see that $(R, r)$ is a cumulant pair on $\mathcal{A}^{0}$. Indeed, if $a_{1}, \ldots, a_{m}$, $a_{p} a_{p+1} \in \mathcal{A}^{0}$ then, by the last lemma, we may assume that $a_{1}, \ldots, a_{m} \in \mathcal{A}_{i}$ for some $i \in I$ (otherwise we have 0 on both sides of (1)) and use the fact that $\left(R_{i}, r_{i}\right)$ is a cumulant pair on $\mathcal{A}_{i}$. Denote by $(F, f)$ the related moment pair.

Lemma 4. Suppose $a_{1} \in \mathcal{A}_{i_{1}}, \ldots, a_{m} \in \mathcal{A}_{i_{m}}, b_{1} \in \mathcal{A}_{j_{1}}, \ldots, b_{n} \in \mathcal{A}_{j_{n}}$ and $a_{1} \ldots a_{m}=b_{1} \ldots b_{n}$. Then $F\left(a_{1}, \ldots, a_{m}\right)=F\left(b_{1}, \ldots, b_{n}\right)$ and $f\left(a_{1}, \ldots, a_{m}\right)$ $=f\left(b_{1}, \ldots, b_{n}\right)$.

Proof. By multiplicativity we can assume that $i_{1} \neq \ldots \neq i_{m}, j_{1} \neq \ldots$ $\neq j_{n}$ and $a_{1}, \ldots, a_{m}, b_{1}, \ldots, b_{n} \neq K 1$. Then $a_{1} \ldots a_{m}=b_{1} \ldots b_{n}$ implies that $m=n$ and $b_{1}=\alpha_{1} a_{1}, \ldots, b_{m}=\alpha_{m} a_{m}$ for some $\alpha_{1}, \ldots, \alpha_{m} \in K$ satisfying $\alpha_{1} \ldots \alpha_{m}=1$, which ends the proof.

The last lemma allows us to define linear functions $\Phi$ and $\psi$ on $\mathcal{A}=$ $*_{i \in I} \mathcal{A}_{i}$ by putting

$$
\Phi\left(a_{1} \ldots a_{m}\right)=F\left(a_{1}, \ldots, a_{m}\right), \quad \psi\left(a_{1} \ldots a_{m}\right)=f\left(a_{1}, \ldots, a_{m}\right)
$$

whenever $a_{1} \in \mathcal{A}_{i_{1}}, \ldots, a_{m} \in \mathcal{A}_{i_{m}}$ for some $i_{1}, \ldots, i_{m} \in I$.

Theorem 1. Assume that $a_{1} \in \mathcal{A}_{i_{1}}, \ldots, a_{m} \in \mathcal{A}_{i_{m}}, m \geq 1, i_{1} \neq \ldots \neq i_{m}$ and $\psi_{i_{1}}\left(a_{1}\right)=\ldots=\psi_{i_{m}}\left(a_{m}\right)=0$. Then

$$
\psi\left(a_{1} \ldots a_{m}\right)=0 \quad \text { and } \quad \Phi\left(a_{1} \ldots a_{m}\right)=\Phi_{i_{1}}\left(a_{1}\right) \ldots \Phi_{i_{m}}\left(a_{m}\right) .
$$

Proof. We proceed by induction on $m$. For $m=1$ the assertion is obvious. Suppose it holds for all $k<m$ and write

$$
\begin{aligned}
\psi\left(a_{1} \ldots a_{m}\right) & =\sum_{\pi \in \mathrm{NC}(m)} \prod_{V \in \pi} r\left(\left(a_{1}, \ldots, a_{m}\right)(V)\right), \\
\Phi\left(a_{1} \ldots a_{m}\right) & =\sum_{\pi \in \mathrm{NC}(m)} \prod_{V \in \pi_{\mathrm{i}}} r\left(\left(a_{1}, \ldots, a_{m}\right)(V)\right) \prod_{V \in \pi_{\circ}} R\left(\left(a_{1}, \ldots, a_{m}\right)(V)\right) .
\end{aligned}
$$


By Lemma 2 and by induction we can take in these sums only partitions satisfying $\pi_{\mathrm{i}}=\emptyset$, i.e. partitions for which all blocks are intervals. But if $V$ is an interval with $|V|>1$ then, by Lemma $3, r\left(\left(a_{1}, \ldots, a_{m}\right)(V)\right)=0$ and $R\left(\left(a_{1}, \ldots, a_{m}\right)(V)\right)=0$. Therefore we are left with $\pi=\{\{1\}, \ldots,\{m\}\}$, which gives the assertion.

The function $\psi$ is called the unital free product of the functions $\psi_{i}$ and denoted by $*_{i \in I} \psi_{i}$ (see $[\mathrm{V}, \mathrm{VDN}]$ ). Hence we will call the pair $(\Phi, \psi)$ of functions on $\mathcal{A}=*_{i \in I} \mathcal{A}_{i}$ the unital free product of the pairs $\left(\Phi_{i}, \psi_{i}\right)$ and denote it by $*_{i \in I}\left(\Phi_{i}, \psi_{i}\right)$. The next proposition says that this operation is associative, which is not the case when we have more than two states (see [M2]).

Proposition 2. Assume that $I=\bigcup_{j \in J} I_{j}$ is a partition of $I$. Then

$$
*_{j \in J}\left(*_{i \in I_{j}}\left(\Phi_{i}, \psi_{i}\right)\right)=*_{i \in I}\left(\Phi_{i}, \psi_{i}\right) .
$$

Proof. Set $\left(\Phi_{j}^{\prime}, \psi_{j}^{\prime}\right)=*_{i \in I_{j}}\left(\Phi_{i}, \psi_{i}\right)$ and $\left(\Phi^{\prime}, \psi^{\prime}\right)=*_{j \in J}\left(\Phi_{j}^{\prime}, \psi_{j}^{\prime}\right)$. Take $a_{1} \in \mathcal{A}_{i_{1}}, \ldots, a_{m} \in \mathcal{A}_{i_{m}}$ with $i_{1} \neq \ldots \neq i_{m}$ and $\psi_{i_{1}}\left(a_{1}\right)=\ldots=\psi_{i_{m}}\left(a_{m}\right)$ $=0$. Then for some $0=k(0)<k(1)<\ldots<k(s)=m$ we have $i_{k(t-1)+1}$, $i_{k(t-1)+2}, \ldots, i_{k(t)} \in I_{j_{t}}, 1 \leq t \leq s$, and $j_{1} \neq \ldots \neq j_{s}$. Put

$$
b_{t}=a_{k(t-1)+1} a_{k(t-1)+2} \ldots a_{k(t)} .
$$

Then we have $\psi_{j_{t}}^{\prime}\left(b_{t}\right)=0$ and

$$
\Phi_{j_{t}}^{\prime}\left(b_{t}\right)=\Phi_{i_{k(t-1)+1}}\left(a_{k(t-1)+1}\right) \Phi_{i_{k(t-1)+2}}\left(a_{k(t-1)+2}\right) \ldots \Phi_{i_{k(t)}}\left(a_{k(t)}\right) .
$$

This in turn implies $\psi^{\prime}\left(a_{1} \ldots a_{m}\right)=\psi^{\prime}\left(b_{1} \ldots b_{s}\right)=0$ and $\Phi^{\prime}\left(a_{1} \ldots a_{m}\right)=$ $\Phi^{\prime}\left(b_{1} \ldots b_{s}\right)=\Phi_{j_{1}}^{\prime}\left(b_{1}\right) \ldots \Phi_{j_{s}}^{\prime}\left(b_{s}\right)=\Phi_{i_{1}}\left(a_{1}\right) \ldots \Phi_{i_{m}}\left(a_{m}\right)$, which concludes the proof.

The following two propositions can help us evaluate $\Phi\left(a_{1} \ldots a_{m}\right)$.

Proposition 3. Assume that $a_{1} \in \mathcal{A}_{i_{1}}, \ldots, a_{m} \in \mathcal{A}_{i_{m}}$ and that for some $1 \leq p<m$ the sets $\left\{i_{1}, \ldots, i_{p}\right\}$ and $\left\{i_{p+1}, \ldots, i_{m}\right\}$ are disjoint. Then

$$
\Phi\left(a_{1} \ldots a_{m}\right)=\Phi\left(a_{1} \ldots a_{p}\right) \Phi\left(a_{p+1} \ldots a_{m}\right) .
$$

Proof. In view of Lemma 3 we can calculate $\Phi\left(a_{1} \ldots a_{m}\right)$ using only $\pi$ 's of the form $\pi=\pi^{1} \pi^{2}$, where $\pi^{1} \in \mathrm{NC}(\{1, \ldots, p\}), \pi^{2} \in \mathrm{NC}(\{p+1, \ldots, m\})$.

Proposition 4. Suppose that $a_{1} \in \mathcal{A}_{i_{1}}, \ldots, a_{m} \in \mathcal{A}_{i_{m}}$ and that for some $k$ we have $i_{k} \notin\left\{i_{1}, \ldots, i_{k-1}, i_{k+1}, \ldots, i_{m}\right\}$. Then

$$
\begin{aligned}
\Phi\left(a_{1} \ldots a_{m}\right)= & \psi_{i_{k}}\left(a_{k}\right) \Phi\left(a_{1} \ldots a_{k-1} a_{k+1} \ldots a_{m}\right) \\
& +\Phi\left(a_{1} \ldots a_{k-1}\right)\left[\Phi_{i_{k}}\left(a_{k}\right)-\psi_{i_{k}}\left(a_{k}\right) \mathbf{1}\right] \Phi\left(a_{k+1} \ldots a_{m}\right) .
\end{aligned}
$$

More generally, if the sets $\left\{i_{p}, i_{p+1}, \ldots, i_{q}\right\}$ and $\left\{i_{1}, \ldots, i_{p-1}, i_{q+1}, \ldots, i_{m}\right\}$ are disjoint for some $1 \leq p \leq q \leq m$ then

$$
\begin{aligned}
\Phi\left(a_{1} \ldots a_{m}\right)= & \psi\left(a_{p} \ldots a_{q}\right) \Phi\left(a_{1} \ldots a_{p-1} a_{q+1} \ldots a_{m}\right) \\
& +\Phi\left(a_{1} \ldots a_{p-1}\right)\left[\Phi\left(a_{p} \ldots a_{q}\right)-\psi\left(a_{p} \ldots a_{q}\right) \mathbf{1}\right] \Phi\left(a_{q+1} \ldots a_{m}\right) .
\end{aligned}
$$


Proof. Assume first that $\psi_{i_{k}}\left(a_{k}\right)=0$ and put $r(V):=r\left(\left(a_{1}, \ldots, a_{m}\right)(V)\right)$, $R(V):=R\left(\left(a_{1}, \ldots, a_{m}\right)(V)\right)$. Since $R(V)=0$ and $r(V)=0$ for every $V$ with $\{k\} \subset V$ and $|V|>1$, and moreover $r(\{k\})=0$, we have

$$
\begin{aligned}
\Phi\left(a_{1} \ldots a_{m}\right)= & \sum_{\pi \in \mathrm{NC}(m)} \prod_{V \in \pi_{\mathrm{i}}} r(V) \prod_{V \in \pi_{\circ}} R(V) \\
= & \sum_{\substack{\pi \in \mathrm{NC}\{1, \ldots, k-1\} \\
\sigma \in \mathrm{NC}\{k+1, \ldots, m\}}}\left(\prod_{V \in \pi_{\mathrm{i}}} r(V) \prod_{V \in \pi_{\circ}} R(V)\right) \Phi_{i_{k}}\left(a_{k}\right) \\
& \cdot\left(\prod_{W \in \sigma_{\mathrm{i}}} r(V) \prod_{V \in \sigma_{\circ}} R(V)\right) \\
& =\Phi\left(a_{1} \ldots a_{k-1}\right) \Phi_{i_{k}}\left(a_{k}\right) \Phi\left(a_{k+1} \ldots a_{m}\right) .
\end{aligned}
$$

Applying this to the general case we have

$$
\begin{aligned}
& \Phi\left(a_{1} \ldots a_{k-1}\left(a_{k}-\psi_{i_{k}}\left(a_{k}\right) 1\right) a_{k+1} \ldots a_{m}\right) \\
& \quad=\Phi\left(a_{1} \ldots a_{k-1}\right)\left[\Phi_{i_{k}}\left(a_{k}\right)-\psi_{i_{k}}\left(a_{k}\right) \mathbf{1}\right] \Phi\left(a_{k+1} \ldots a_{m}\right),
\end{aligned}
$$

which proves the first assertion. Combining this with Proposition 2 we get the second assertion.

5. Limit theorems. In this section we assume that $\mathcal{A}$ is a fixed complex unital algebra with linear functions $\Phi: \mathcal{A} \rightarrow \mathcal{B}, \psi: \mathcal{A} \rightarrow \mathbb{C}$, where $\mathcal{B}$ is a complex algebra with a unit $\mathbf{1}$ and with a norm $\|\cdot\|$, satisfying $\Phi(1)=\mathbf{1}$ and $\psi(1)=1$, and that $(R, r)$ is the related cumulant pair on $\mathcal{A}$. Define $(\widehat{\mathcal{A}}, \widehat{\Phi}, \widehat{\psi})=*_{i \in \mathbb{N}}(\mathcal{A}, \Phi, \psi)$. For $a \in \mathcal{A}, i \in \mathbb{N}$, we denote by $(a, i)$ the embedding of $a$ into the $i$ th factor $\mathcal{A}$ of $\widehat{\mathcal{A}}$. For example $\left(a_{1}, i\right) \cdot\left(a_{2}, i\right)=\left(a_{1} a_{2}, i\right)$. The following results generalise those presented in [S1], [BS] and [BLS].

Theorem 2. Let $m \geq 1$ be a fixed integer and assume that for every $N \in \mathbb{N}$ elements $a_{1, N}, a_{2, N}, \ldots, a_{m, N} \in \mathcal{A}$ are given. Assume that for every nonempty subset $V \subset\{1, \ldots, m\}$ the limits

$$
\lim _{N \rightarrow \infty} N \cdot \Phi\left(\prod_{k \in V} a_{k, N}\right)=Q(V), \quad \lim _{N \rightarrow \infty} N \cdot \psi\left(\prod_{k \in V} a_{k, N}\right)=q(V)
$$

exist. Set

$$
S_{k, N}=\left(a_{k, N}, 1\right)+\left(a_{k, N}, 2\right)+\ldots+\left(a_{k, N}, N\right) .
$$

Then

$$
\lim _{N \rightarrow \infty} \widehat{\Phi}\left(S_{1, N} S_{2, N} \ldots S_{m, N}\right)=\sum_{\pi \in \mathrm{NC}(m)} \prod_{V \in \pi_{\mathrm{i}}} q(V) \prod_{V \in \pi_{\circ}} Q(V)
$$

and

$$
\lim _{N \rightarrow \infty} \widehat{\psi}\left(S_{1, N} S_{2, N} \ldots S_{m, N}\right)=\sum_{\pi \in \mathrm{NC}(m)} \prod_{V \in \pi} q(V)
$$


Proof. By induction on $m$ and by Proposition 1 we see that if $\emptyset \neq V \subset$ $\{1, \ldots, m\}$ then

$$
\begin{aligned}
& \lim _{N \rightarrow \infty} N \cdot R\left(\left(a_{1, N}, \ldots, a_{m, N}\right)(V)\right)=Q(V), \\
& \lim _{N \rightarrow \infty} N \cdot r\left(\left(a_{1, N}, \ldots, a_{m, N}\right)(V)\right)=q(V) .
\end{aligned}
$$

Now consider

$$
\widehat{\Phi}\left(S_{1, N} \ldots S_{m, N}\right)=\sum_{i(1), \ldots, i(m) \in\{1, \ldots, N\}} \widehat{\Phi}\left(\left(a_{1, N}, i(1)\right) \ldots\left(a_{m, N}, i(m)\right)\right) .
$$

For a sequence $\mathbf{i}=(i(1), \ldots, i(m)) \in\{1, \ldots, N\}^{m}$ we define a partition $\pi=\pi(\mathbf{i})$ of $\{1, \ldots, m\}$ by $k \stackrel{\pi}{\sim} l$ iff $i(k)=i(l)$. Note that if $\pi(\mathbf{i})=\pi(\mathbf{j}):=\pi$ then

$$
\widehat{\Phi}\left(\left(a_{1, N}, i(1)\right) \ldots\left(a_{m, N}, i(m)\right)\right)=\widehat{\Phi}\left(\left(a_{1, N}, j(1)\right) \ldots\left(a_{m, N}, j(m)\right)\right) .
$$

Denoting this common value by $\widehat{\Phi}(\pi ; N)$ we note that if $|\pi|=p$ then there are exactly $A(p, N):=N(N-1) \ldots(N-p+1)$ sequences $\mathbf{i} \in\{1, \ldots, N\}^{m}$ with $\pi(\mathbf{i})=\pi$. Hence

$$
\widehat{\Phi}\left(S_{1, N} \ldots S_{m, N}\right)=\sum_{\pi} A(|\pi|, N) \widehat{\Phi}(\pi ; N),
$$

where the sum is taken over all partitions $\pi$ of $\{1, \ldots, m\}$. For fixed $\pi$ we have

$$
\begin{aligned}
& \widehat{\Phi}(\pi ; N) \\
& =\sum_{\substack{\sigma \in \mathrm{NC}(m) \\
\sigma \leq \pi}} \prod_{B \in \sigma_{\mathrm{i}}} r\left(\left(a_{1, N}, \ldots, a_{m, N}\right)(B)\right) \prod_{B \in \sigma_{\circ}} R\left(\left(a_{1, N}, \ldots, a_{m, N}\right)(B)\right),
\end{aligned}
$$

where $\sigma \leq \pi$ means that $\sigma$ is a subpartition of $\pi$, i.e. every block of $\sigma$ is a subset of a block of $\pi$. Note that every summand is a product of $|\sigma|$ factors. Therefore

$$
\lim _{N \rightarrow \infty} A(|\pi|, N) \cdot \widehat{\Phi}(\pi ; N)=\prod_{V \in \pi_{\mathrm{i}}} q(V) \prod_{V \in \pi_{\mathrm{o}}} Q(V)
$$

if $\pi$ is noncrossing, and the limit is 0 otherwise. The same proof works for $\psi$.

Denote by $\mathrm{NC}_{2}(m)$ the class of all partitions $\pi \in \mathrm{NC}(m)$ satisfying $|V|=2$ for every block $V \in \pi$. Of course if $m$ is odd then $\mathrm{NC}_{2}(m)$ is empty and a sum over $\pi \in \mathrm{NC}_{2}(m)$ equals 0 .

Corollary 1 (Conditionally free central limit theorem). Let $a_{1}, \ldots, a_{m}$ $\in \mathcal{A}$ with $\Phi\left(a_{k}\right)=0, \psi\left(a_{k}\right)=0$ for $1 \leq k \leq m$ and set

$$
S_{k, N}=\frac{1}{\sqrt{N}}\left[\left(a_{k}, 1\right)+\ldots+\left(a_{k}, N\right)\right] .
$$


Then, for $m$ even,

$$
\lim _{N \rightarrow \infty} \widehat{\Phi}\left(S_{1, N} \ldots S_{m, N}\right)=\sum_{\pi \in \mathrm{NC}_{2}(m)} \prod_{\substack{V \in \pi_{\mathrm{i}} \\ V=\{k, l\}, k<l}} \psi\left(a_{k} a_{l}\right) \prod_{\substack{V \in \pi_{\circ} \\ V=\{k, l\}, k<l}} \Phi\left(a_{k} a_{l}\right)
$$

and

$$
\lim _{N \rightarrow \infty} \widehat{\psi}\left(S_{1, N} \ldots S_{m, N}\right)=\sum_{\pi \in \mathrm{NC}_{2}(m)} \prod_{\substack{V \in \pi \\ V=\{k, l\}, k<l}} \psi\left(a_{k} a_{l}\right),
$$

and both limits are 0 if $m$ is odd.

Proof. Putting $a_{k, N}=(1 / \sqrt{N}) a_{k}$ we have $\Phi\left(a_{k, N}\right)=0, \psi\left(a_{k, N}\right)=0$, $N \cdot \Phi\left(a_{k, N} a_{l, N}\right)=\Phi\left(a_{k} a_{l}\right), N \cdot \psi\left(a_{k, N} a_{l, N}\right)=\psi\left(a_{k} a_{l}\right)$ and for $V \subset\{1, \ldots, m\}$ with $|V| \geq 3, \lim _{N \rightarrow \infty} N \cdot \Phi\left(\prod_{k \in V} a_{k, N}\right)=0$ and $\lim _{N \rightarrow \infty} N \cdot \psi\left(\prod_{k \in V} a_{k, N}\right)$ $=0$.

Remark. Assume that $\mathcal{B}$ is the algebra $\mathcal{B}\left(\mathcal{H}_{0}\right)$ of bounded linear operators on a Hilbert space $\mathcal{H}_{0}, a_{1}=\ldots=a_{m}=a$, and $\Phi\left(a^{2}\right)=A^{2}$, $0 \leq A \in \mathcal{B}\left(\mathcal{H}_{0}\right), \psi\left(a^{2}\right)=\beta^{2}, \beta>0$. We know from [BLS] that for $\alpha>0$ the unique probability measure $\nu_{\alpha, \beta}$ on $\mathbb{R}$ satisfying

$$
\int t^{m} d \nu_{\alpha, \beta}(t)=\sum_{\pi \in \mathrm{NC}_{2}(m)} \alpha^{2\left|\pi_{\circ}\right|} \beta^{2\left|\pi_{\mathrm{i}}\right|}
$$

for every $m$ is given by

$$
\nu_{\alpha, \beta}=c(\alpha, \beta)\left(\delta_{\alpha^{2} / \sqrt{\alpha^{2}-\beta^{2}}}+\delta_{-\alpha^{2} / \sqrt{\alpha^{2}-\beta^{2}}}\right)+\widetilde{\nu}_{\alpha, \beta},
$$

where

$$
c(\alpha, \beta)= \begin{cases}\frac{1}{2} \frac{\alpha^{2}-2 \beta^{2}}{\alpha^{2}-\beta^{2}} & \text { if } 2 \beta^{2} \leq \alpha^{2}, \\ 0 & \text { otherwise }\end{cases}
$$

(note that $[\mathrm{BLS}]$ erroneously had " $\frac{1}{4}$ " instead of “ $\frac{1}{2}$ "),

$$
d \widetilde{\nu}_{\alpha, \beta}=\chi_{[-2 \beta, 2 \beta]}(t) \frac{1}{2 \pi} \frac{\alpha^{2} \sqrt{4 \beta^{2}-t^{2}}}{\alpha^{4}-\left(\alpha^{2}-\beta^{2}\right) t^{2}} d t .
$$

For fixed $\alpha, \beta>0$ and for a continuous function $f: \mathbb{R} \rightarrow \mathbb{R}$, define

$$
C(f ; \alpha, \beta)=C_{1}(f ; \alpha, \beta)+C_{2}(f ; \alpha, \beta),
$$

where

$$
\begin{aligned}
& C_{1}(f ; \alpha, \beta)=\frac{1}{2} \frac{\alpha^{2}-2 \beta^{2}}{\alpha^{2}-\beta^{2}}\left(f\left(\frac{\alpha^{2}}{\sqrt{\alpha^{2}-\beta^{2}}}\right)+f\left(\frac{-\alpha^{2}}{\sqrt{\alpha^{2}-\beta^{2}}}\right)\right) \chi_{[\beta \sqrt{2}, \infty)}(\alpha) \\
& C_{2}(f ; \alpha, \beta)=\frac{1}{2 \pi} \int_{-2 \beta}^{2 \beta} f(t) \frac{\alpha^{2} \sqrt{4 \beta^{2}-t^{2}}}{\alpha^{4}-\left(\alpha^{2}-\beta^{2}\right) t^{2}} d t .
\end{aligned}
$$


Now taking the spectral resolution $A=\int_{0}^{\infty} \alpha d E(\alpha)$ of $A$ we can describe our operator-valued limit measure, which has moments

$$
\int t^{m} d \nu_{A, \beta}(t)=\sum_{\pi \in \mathrm{NC}_{2}(m)} \beta^{2\left|\pi_{\mathrm{i}}\right|} A^{2\left|\pi_{\mathrm{o}}\right|}
$$

by

$$
\int f(t) d \nu_{A, \beta}(t)=\int_{0}^{\infty} C(f ; \alpha, \beta) d E(\alpha) .
$$

Corollary 2 (Conditionally free Poisson limit theorem). Assume that $a_{1}, a_{2}, \ldots \in \mathcal{A}$ with

$$
\lim _{N \rightarrow \infty} N \cdot \Phi(\underbrace{a_{N} \ldots a_{N}}_{s \text { times }})=A, \quad \lim _{N \rightarrow \infty} N \cdot \psi(\underbrace{a_{N} \ldots a_{N}}_{s \text { times }})=\beta
$$

for every $s$ with $1 \leq s \leq m$. Then for

$$
S_{N}=\left(a_{N}, 1\right)+\ldots+\left(a_{N}, N\right)
$$

we have

$$
\begin{aligned}
& \lim _{N \rightarrow \infty} \widehat{\Phi}(\underbrace{S_{N} \ldots S_{N}}_{m \text { times }})=\sum_{\pi \in \mathrm{NC}(m)} \beta^{\left|\pi_{\mathrm{i}}\right|} A^{\left|\pi_{\mathrm{o}}\right|}, \\
& \lim _{N \rightarrow \infty} \widehat{\psi}(\underbrace{S_{N} \ldots S_{N}}_{m \text { times }})=\sum_{\pi \in \mathrm{NC}(m)} \beta^{|\pi|} .
\end{aligned}
$$

Proof. For every $\emptyset \neq V \subset\{1, \ldots, m\}$ we have $q(V)=\beta$ and $Q(V)=A$.

REMARK. As before, we can apply the formula provided in [BLS] to find that, in the case of an operator $0 \leq A \in \mathcal{B}\left(\mathcal{H}_{0}\right)$ with spectral resolution $A=\int_{0}^{\infty} \alpha d E(\alpha)$, the unique operator-valued probability measure on $\mathbb{R}$ with moments

$$
\int t^{m} d \pi_{A, \beta}(t)=\sum_{\pi \in \mathrm{NC}(m)} \beta^{\left|\pi_{\mathrm{i}}\right|} A^{\left|\pi_{\mathrm{o}}\right|}
$$

is given by

$$
\int_{-\infty}^{\infty} f(t) d \pi_{A, \beta}=\int_{0}^{\infty} P(f ; \alpha, \beta) d E(\alpha)
$$

where

$$
\begin{aligned}
P(f ; \alpha, \beta) & =P_{1}(f ; \alpha, \beta)+P_{2}(f ; \alpha, \beta)+P_{3}(f ; \alpha, \beta), \\
P_{1}(f ; \alpha, \beta) & =\frac{1-\beta}{1+\alpha-\beta} f(0) \cdot \chi_{[0,1]}(\beta), \\
P_{2}(f ; \alpha, \beta) & =\frac{(\alpha-\beta-\sqrt{\beta})(\alpha-\beta+\sqrt{\beta})}{(\alpha-\beta)(\alpha-\beta+1)} f\left(\frac{\alpha(\alpha-\beta+1)}{\alpha-\beta}\right) \cdot \chi_{\beta}(\alpha),
\end{aligned}
$$


$\chi_{\beta}$ denotes the characteristic function of the set $[0, \max \{0, \beta-\sqrt{\beta}\}] \cup$ $[\beta+\sqrt{\beta}, \infty)$, and

$$
P_{3}(f ; \alpha, \beta)=\frac{1}{\pi} \int_{1+\beta-2 \sqrt{\beta}}^{1+\beta+2 \sqrt{\beta}} f(t) \frac{\alpha \sqrt{4 \beta-(t-(1+\beta))^{2}}}{2 t[t(\beta-\alpha)+\alpha(1-\beta+\alpha)]} d t .
$$

6. Free product of pairs of representations. Let $\mathcal{A}$ be a complex unital *-algebra. We will call a functional $\psi$ on $\mathcal{A}$ a state if $\psi$ is of the form $\psi(a)=\langle\sigma(a) \xi, \xi\rangle$ for a $*$-representation $\sigma$ of $\mathcal{A}$ in a Hilbert space $\mathcal{K}$ and a unit vector $\xi \in \mathcal{K}$. More generally, if $\mathcal{H}_{0}$ is a Hilbert space then an operator-valued function $\Phi: \mathcal{A} \rightarrow \mathcal{B}\left(\mathcal{H}_{0}\right)$ will be called a state if $\Phi$ is of the form $\Phi(a)=\left.P_{0} \pi(a)\right|_{\mathcal{H}_{0}}$, where $\pi$ is a $*$-representation of $\mathcal{A}$ in a Hilbert space $\mathcal{H} \supseteq \mathcal{H}_{0}$ and $P_{0}$ is the orthogonal projection from $\mathcal{H}$ onto $\mathcal{H}_{0}$. In particular the functions $\Phi$ and $\psi$ are completely positive. We are going to show that free product of pairs of states is a pair of states. For this purpose we generalize Voiculescu's construction of the free product representation (see $[\mathrm{V}, \mathrm{VDN}])$.

Theorem 3. Assume that for every $i \in I, \Phi_{i}$ and $\psi_{i}$ are states on a complex unital algebra $\mathcal{A}_{i}$. Then $(\Phi, \psi)=*_{i \in I}\left(\Phi_{i}, \psi_{i}\right)$ is a pair of states on $\mathcal{A}=*_{i \in I} \mathcal{A}_{i}$.

Proof. For $i \in I$ assume that $\psi_{i}(a)=\left\langle\sigma_{i}(a) \xi_{i}, \xi_{i}\right\rangle$ and $\Phi_{i}(a)=\left.P_{0}^{i} \pi_{i}(a)\right|_{\mathcal{H}_{0}}$, $a \in \mathcal{A}_{i}$ where $\pi_{i}: \mathcal{A}_{i} \rightarrow \mathcal{B}\left(\mathcal{H}_{0} \oplus \mathcal{H}_{i}\right), \sigma_{i}: \mathcal{A}_{i} \rightarrow \mathcal{B}\left(\mathbb{C} \xi_{i} \oplus \mathcal{K}_{i}\right)$ are *representations of $\mathcal{A}_{i}$ and $P_{0}^{i}$ is the orthogonal projection of $\mathcal{H}_{0} \oplus \mathcal{H}_{i}$ onto $\mathcal{H}_{0}$. Now define

$$
\mathcal{H}=\mathcal{H}_{0} \oplus \bigoplus_{m=1}^{\infty}\left(\bigoplus_{\substack{i_{1}, \ldots, i_{m} \in I \\ i_{1} \neq \ldots \neq i_{m}}} \mathcal{K}_{i_{1}} \otimes \ldots \otimes \mathcal{K}_{i_{m-1}} \otimes \mathcal{H}_{i_{m}}\right) .
$$

For each $i \in I$ we have the decomposition

$$
\mathcal{H}=\left(\mathcal{H}_{0} \oplus \mathcal{H}_{i}\right) \oplus\left(\mathbb{C} \xi_{i} \oplus \mathcal{K}_{i}\right) \otimes \mathcal{H}(i),
$$

where

$$
\mathcal{H}(i)=\bigoplus_{m=1}^{\infty}\left(\bigoplus_{\substack{i_{1}, \ldots, i_{m} \in I \\ i \neq i_{1} \neq \ldots \neq i_{m}}} \mathcal{K}_{i_{1}} \otimes \ldots \otimes \mathcal{K}_{i_{m-1}} \otimes \mathcal{H}_{i_{m}}\right) .
$$

Then we define a $*$-representation $\tilde{\pi}_{i}$ of $\mathcal{A}_{i}$ on $\mathcal{H}$ to act as $\pi_{i}(a)$ on $\mathcal{H}_{0} \oplus \mathcal{H}_{i}$ and as $\sigma_{i}(a) \otimes \operatorname{Id}_{\mathcal{H}(i)}$ on $\left(\mathbb{C} \xi_{i} \oplus \mathcal{K}_{i}\right) \otimes \mathcal{H}(i)$. Having $\tilde{\pi}_{i}$ defined for each $i \in I$ we define a representation $\pi$ of $\mathcal{A}=*_{i \in I} \mathcal{A}_{i}$ by putting $\pi(a)=$ $\widetilde{\pi}_{i_{1}}\left(a_{1}\right) \ldots \widetilde{\pi}_{i_{m}}\left(a_{m}\right)$ for $a=a_{1} \ldots a_{m}, a_{k} \in \mathcal{A}_{i_{k}}, i_{1} \neq \ldots \neq i_{m}$. Obviously, $\pi$ is a $*$-representation of $\mathcal{A}$. We are going to show that $\Phi(a)=\left.P_{0} \pi(a)\right|_{\mathcal{H}_{0}}$ for every $a \in \mathcal{A}$. 
Assume that $a_{1} \in \mathcal{A}_{i_{1}}, \ldots, a_{m} \in \mathcal{A}_{i_{m}}, i_{1} \neq \ldots \neq i_{m}$ and $\psi_{i_{1}}\left(a_{1}\right)=\ldots=$ $\psi_{i_{m}}\left(a_{m}\right)=0$. The last condition implies that $\sigma_{i_{k}}\left(a_{k}\right) \xi_{i_{k}} \in \mathcal{K}_{i_{k}}$ and one can check by induction that for $\xi \in \mathcal{H}_{0}$,

$$
\begin{aligned}
\pi\left(a_{1} \ldots a_{m}\right) \xi= & \Phi_{i_{1}}\left(a_{1}\right) \ldots \Phi_{i_{m}}\left(a_{m}\right) \xi \\
& +\sum_{k=1}^{m} \sigma_{i_{1}}\left(a_{1}\right) \xi_{i_{1}} \otimes \ldots \otimes \sigma_{i_{k-1}}\left(a_{k-1}\right) \xi_{i_{k-1}} \\
& \otimes\left(\operatorname{Id}-P_{0}^{i_{k}}\right) \pi_{i_{k}}\left(a_{k}\right) \Phi_{i_{k+1}}\left(a_{k+1}\right) \ldots \Phi_{i_{m}}\left(a_{m}\right) \xi .
\end{aligned}
$$

which implies that $\left.P_{0} \pi\left(a_{1} \ldots a_{m}\right)\right|_{\mathcal{H}_{0}}=\Phi_{i_{1}}\left(a_{1}\right) \ldots \Phi_{i_{m}}\left(a_{m}\right)$.

Now we can repeat this construction replacing $\mathcal{H}_{0}$ by $\mathbb{C} 1, \mathcal{H}_{i}$ by $\mathcal{K}_{i}$ and $\pi_{i}$ by $\sigma_{i}$ to obtain the $*$-representation $\sigma:=*_{i \in I} \sigma_{i}$ corresponding to $\psi$.

REMARK. It is natural to call the pair $(\pi, \sigma)$ the conditionally free prod$u c t$ of the pairs $\left(\pi_{i}, \sigma_{i}\right)$ and to write $(\pi, \sigma):=*_{i \in I}\left(\pi_{i}, \sigma_{i}\right)$. Note that if $\mathcal{A}_{i}$ are $C^{*}$-algebras then the states $\Phi$ and $\psi$ can be extended to ones on the unital $C^{*}$-free product (see [VDN]).

7. Free convolution of pairs of measures. Let $\mathcal{H}$ be a fixed Hilbert space and let $B(\mathcal{H})_{+}$denote the class of all nonnegative bounded linear operators on $\mathcal{H}$. A function $\mu$ defined on the Borel field $\mathcal{B}(\mathbb{R})$ and with values in $B(\mathcal{H})_{+}$is said to be an operator-valued measure if for every $\xi \in \mathcal{H}$ the map $\mathcal{B}(\mathbb{R}) \ni E \mapsto\langle\mu(E) \xi, \xi\rangle$ is a measure (see [Sm] for instance). We call $\mu$ a probability measure if $\mu(\mathbb{R})=\mathrm{Id}$, and we call $\mu$ compactly supported if for some compact set $K \subset \mathbb{R}$ we have $\mu(E)=0$ whenever $E \cap K=\emptyset$. We will denote by $\mathcal{M}(\mathcal{H})$ the set of all compactly supported operator-valued probability measures $\mu: \mathcal{B}(\mathbb{R}) \rightarrow B(\mathcal{H})_{+}$. In particular we define $\Delta_{0} \in$ $\mathcal{M}(\mathcal{H})$ by putting $\Delta_{0}(E)=$ Id if $0 \in E$ and $\Delta_{0}(E)=0$ otherwise. We will denote by $\mathcal{M}$ the class of ordinary compactly supported probability measures on $\mathbb{R}$.

We define the moment sequence $\{(A(m), a(m))\}_{m=0}^{\infty}$ of a pair $(\mu, \nu) \in$ $\mathcal{M}(\mathcal{H}) \times \mathcal{M}$ by

$$
A(m)=\int_{\mathbb{R}} x^{m} d \mu(x), \quad a(m)=\int_{\mathbb{R}} x^{m} d \nu(x)
$$

(note that the sequences $A(m), a(m)$ uniquely determine the measures $\mu, \nu$; see $[\mathrm{Bi}])$. This, in turn, defines the cumulant sequence $\{(R(m), r(m))\}_{m=1}^{\infty}$ of $(\mu, \nu)$ by the relations

$$
a(m)=\sum_{\pi \in \mathrm{NC}(m)} \prod_{V \in \pi} r(|V|), \quad A(m)=\sum_{\pi \in \mathrm{NC}(m)} \prod_{V \in \pi_{\mathrm{i}}} r(|V|) \prod_{V \in \pi_{\mathrm{o}}} R(|V|),
$$

where, as usual, the factors in the product $\prod_{V \in \pi_{\mathrm{o}}} R(|V|)$ are in the same order as the outer blocks of $\pi$. 
TheOREM 4. Assume that $\left\{\left(R_{k}(m), r_{k}(m)\right)\right\}_{m=1}^{\infty}$ is the cumulant sequence of a pair $\left(\mu_{k}, \nu_{k}\right) \in \mathcal{M}(\mathcal{H}) \times \mathcal{M}$ for $k \in\{1,2\}$. Then there exists a unique pair $(\mu, \nu) \in \mathcal{M}(\mathcal{H}) \times \mathcal{M}$ whose cumulant sequence $\{(R(m), r(m))\}_{m=1}^{\infty}$ satisfies

$$
R(m)=R_{1}(m)+R_{2}(m), \quad r(m)=r_{1}(m)+r_{2}(m) .
$$

Proof. Take unital $C^{*}$-algebras $\mathcal{A}_{1}, \mathcal{A}_{2}$, states $\Phi_{k}: \mathcal{A}_{k} \rightarrow \mathcal{B}(\mathcal{H}), \psi_{k}:$ $\mathcal{A}_{k} \rightarrow \mathbb{C}$ and elements $X_{k}=X_{k}^{*} \in \mathcal{A}_{k}$ satisfying $\Phi_{k}\left(X_{k}^{m}\right)=A_{k}(m)$, $\psi_{k}\left(X_{k}^{m}\right)=a_{k}(m)$ and take the $C^{*}$-unital free product $(\mathcal{A}, \Phi, \psi)=\left(\mathcal{A}_{1}, \Phi_{1}, \psi_{1}\right)$ $*\left(\mathcal{A}_{2}, \Phi_{2}, \psi_{2}\right)$. Let $\left(\widetilde{R}_{k}, \widetilde{r}_{k}\right)$ and $(\widetilde{R}, \widetilde{r})$ denote the corresponding cumulant pairs on $\mathcal{A}_{k}$ and on $\mathcal{A}^{0}=\mathcal{A}_{1}+\mathcal{A}_{2} \subset \mathcal{A}$ respectively, so that

$$
\widetilde{R}_{k}(\underbrace{X_{k}, \ldots, X_{k}}_{m \text { times }})=R_{k}(m), \quad \widetilde{r}_{k}(\underbrace{X_{k}, \ldots, X_{k}}_{m \text { times }})=r_{k}(m) .
$$

By the definition of $\widetilde{R}, X_{1}+X_{2} \in \mathcal{A}^{0}$ and

$$
\begin{aligned}
& \widetilde{R}(\underbrace{X_{1}+X_{2}, \ldots, X_{1}+X_{2}}_{m \text { times }})=R_{1}(m)+R_{2}(m), \\
& \widetilde{r}(\underbrace{X_{1}+X_{2}, \ldots, X_{1}+X_{2}}_{m \text { times }})=r_{1}(m)+r_{2}(m) .
\end{aligned}
$$

Then $(\mu, \nu)$ is the unique pair in $\mathcal{M}(\mathcal{H}) \times \mathcal{M}$ satisfying

$$
\Phi\left(\left(X_{1}+X_{2}\right)^{m}\right)=\int_{\mathbb{R}} x^{m} d \mu(x), \quad \psi\left(\left(X_{1}+X_{2}\right)^{m}\right)=\int_{\mathbb{R}} x^{m} d \nu(x) .
$$

Denote the pair $(\mu, \nu)$ in the theorem by $\left(\mu_{1}, \nu_{1}\right) \oplus\left(\mu_{2}, \nu_{2}\right)$. Hence we get a binary operation $\oplus$ on $\mathcal{M}(\mathcal{H}) \times \mathcal{M}$ which is associative and commutative, with $\left(\Delta_{0}, \delta_{0}\right)$ as the neutral element. It is easy to see that $\nu$ is the free convolution of $\nu_{1}$ and $\nu_{2}$ in the sense of Voiculescu (cf. [S2, S3]).

Now for $\mu \in \mathcal{M}(\mathcal{H})$, with the moment sequence $A(m)$, we define its boolean cumulant sequence $\widetilde{R}_{m}, m=1,2, \ldots$, by the following relations:

$$
A(m)=\sum_{\substack{\pi \in \mathrm{NC}(m) \\ \pi_{\mathrm{i}}=\emptyset}} \prod_{V \in \pi} \widetilde{R}(|V|),
$$

where the order of factors corresponds to that of blocks. Note that for the pair $\left(\mu, \delta_{0}\right)$ the cumulant sequence is $\{(\widetilde{R}(m), 0)\}_{m=1}^{\infty}$. This leads to the following

Corollary 3. Assume that for $k \in\{1,2\}$ we have the boolean cumulant sequence $\widetilde{R}_{k}(m)$ of a measure $\mu_{k} \in \mathcal{M}(\mathcal{H})$. Then there exists a unique $\mu \in$ $\mathcal{M}(\mathcal{H})$ whose boolean cumulant sequence $\widetilde{R}(m)$ satisfies $\widetilde{R}(m)=\widetilde{R}_{1}(m)+$ $\widetilde{R}_{2}(m)$. 
Following [SW] we call the measure $\mu$ the boolean convolution of $\mu_{1}$ and $\mu_{2}$ and denote it by $\mu_{1} \uplus \mu_{2}$. This binary operation on $\mathcal{M}(\mathcal{H})$ is associative and commutative with $\Delta_{0}$ as the neutral element. It gains in interest in view of the following

ExAMPLE. Let $E$ and $F$ be the spectral measures of selfadjoint operators $A$ and $B$ respectively. It is easy to see what are the moment sequences:

$$
A(m)=\int \lambda^{m} d E(\lambda)=A^{m}, \quad B(m)=B^{m}
$$

and the cumulant sequences are:

$$
\widetilde{R}(m)=\left\{\begin{array}{ll}
A & \text { if } m=1, \\
0 & \text { otherwise, }
\end{array} \quad \widetilde{S}(m)= \begin{cases}B & \text { if } m=1 \\
0 & \text { otherwise }\end{cases}\right.
$$

This implies that $E \uplus F$ is the spectral measure of the sum $A+B$.

\section{References}

[Bi] T. Bisgaard, Positive definite operator sequences, Proc. Amer. Math. Soc. 121 (1994), 1185-1191.

[B1] M. Bożejko, Positive definite functions on the free group and the noncommutative Riesz product, Boll. Un. Mat. Ital. A (6) 4 (1986), 13-21.

[B2] -, Uniformly bounded representations of free groups, J. Reine Angew. Math. 377 (1987), 170-186.

[B3] - Positive-definite kernels, length functions on groups and noncommutative von Neumann inequality, Studia Math. 95 (1989), 107-118.

[BS] M. Bożejko and R. Speicher, $\psi$-independent and symmetrized white noises, in: Quantum Probability and Related Topics, Vol. VI, L. Accardi (ed.), World Sci., 1991, 219-236.

[BLS] M. Bożejko, M. Leinert and R. Speicher, Convolution and limit theorems for conditionally free random variables, Pacific J. Math. 175 (1996), 357-388.

[M1] W. Młotkowski, Free product of representations, in: Contemp. Math. 206, Amer. Math. Soc., 1987, 145-148.

[M2] - Free probability on algebras with infinitely many states, Probab. Theory Related Fields 115 (1999), 579-596.

[Sm] K. Schmüdgen, On a generalization of the classical moment problem, J. Math. Anal. Appl. 125 (1987), 461-470.

[Sh] D. Shlyakhtenko, Random Gaussian band matrices and freeness with amalgamation, Internat. Math. Res. Notices 1996, no. 20, 1013-1025.

[S1] R. Speicher, A new example of "independence" and "white noise", Probab. Theory Related Fields 84 (1990), 141-159.

[S2] - Multiplicative functions on the lattice of non-crossing partitions and free convolution, Math. Ann. 298 (1994), 611-628.

[S3] - Combinatorial theory of the free product with amalgamation and operatorvalued free probability theory, Mem. Amer. Math. Soc. 627 (1998).

[SW] R. Speicher and R. Woroudi, Boolean convolution, Fields Inst. Comm. 12 (1997), $267-279$. 
[V] D. Voiculescu, Symmetries of some reduced free product $C^{*}$-algebras, in: Operator Algebras and their Connections with Topology and Ergodic Theory (Buşteni, 1983), Lecture Notes in Math. 1132, Springer, 1985, 556-588.

[VDN] D. Voiculescu, K. J. Dykema and A. Nica, Free Random Variables, CRM Monograph Ser. 1, Amer. Math. Soc., 1992.

\author{
Mathematical Institute \\ University of Wrocław \\ Pl. Grunwaldzki 2/4 \\ 50-384 Wrocław, Poland \\ E-mail: mlotkow@math.uni.wroc.pl
}

Received August 7, 2000

Revised version April 10, 2002 